\title{
Increased platelet count and leucocyte-platelet complex formation in acute symptomatic compared with asymptomatic severe carotid stenosis
}

\author{
D J H McCabe, P Harrison, I J Mackie, P S Sidhu, G Purdy, A S Lawrie, H Watt, S J Machin, \\ M M Brown
}

J Neurol Neurosurg Psychiatry 2005;76:1249-1254. doi: 10.1136/jnnp.2004.051003

See end of article for authors' affiliations .....................

Correspondence to: Dr D J H McCabe, University Department of Clinical Neurosciences, Royal Free and University College Medical School, The Royal Free Hospital Hampstead, Pond St, London NW3 2PF, England; d.mccabe@ medsch.ucl.ac.uk

Received 31 July 2004 Revised version received 27 October 2004 Accepted 4December 2004

\begin{abstract}
Objective: The risk of stroke in patients with recently symptomatic carotid stenosis is considerably higher than in patients with asymptomatic stenosis. In the present study it was hypothesised that excessive platelet activation might partly contribute to this difference.

Methods: A full blood count was done and whole blood flow cytometry used to measure platelet surface expression of $C D 62 \mathrm{P}, \mathrm{CD} 63$, and $\mathrm{PAC1}$ binding and the percentage of leucocyte-platelet complexes in patients with acute $(0-21$ days, $n=19)$ and convalescent (79-365 days) symptomatic $(n=16)$ and asymptomatic $(n=16)$ severe $(\geqslant 70 \%)$ carotid stenosis. Most patients were treated with aspirin (37.5$300 \mathrm{mg}$ daily) although alternative antithrombotic regimens were more commonly used in the symptomatic group.

Results: The mean platelet count was higher in patients with acute and convalescent symptomatic compared with asymptomatic carotid stenosis. There were no significant differences in the median percentage expression of CD62P and CD63, or PAC1 binding between the acute or convalescent symptomatic and asymptomatic patients. The median percentages of neutrophil-platelet $(p=0.004)$, monocyte-platelet $(p=0.046)$, and lymphocyte-platelet complexes $(p=0.02)$ were higher in acute symptomatic than in asymptomatic patients. In patients on aspirin monotherapy, the percentages of neutrophil-platelet and monocyte-platelet complexes $(p=0.03)$ were higher in acute symptomatic $(n=11)$ than asymptomatic patients $(n=14)$. In the convalescent phase, the median percentages of all leucocyte-platelet complexes in the symptomatic group dropped to levels similar to those found in the asymptomatic group.

Conclusion: Increased platelet count and leucocyte-platelet complex formation may contribute to the early excess risk of stroke in patients with recently symptomatic carotid stenosis.
\end{abstract}

$\mathrm{T}$ he two year risk of stroke in the arterial territory supplied by a severely stenosed $(\geqslant 70 \%)$ internal carotid artery is approximately $4 \%$ in asymptomatic patients in whom the stenosis is incidentally picked up. ${ }^{12}$ However, this rate rises to $26 \%$ in patients with recent stroke or transient ischaemic attack (TIA) who are treated with medical therapy alone. ${ }^{3}$ This risk is highest in the first few weeks after the onset of symptoms. The mechanisms responsible for this disparity in the risk of stroke between patients with asymptomatic and symptomatic severe carotid stenosis are not fully understood. Differences in the morphology and/or stability of the atherosclerotic plaque, ${ }^{4}$ different degrees of endothelial activation, or differences in thrombogenicity of the circulating blood could play a role. Because platelets play a pivotal role in thromboembolism, excessive platelet activation could contribute to the higher risk of stroke in patients with symptomatic carotid stenosis. However, comparative histological studies have not consistently shown a higher incidence of luminal thrombus on the surface of symptomatic compared with asymptomatic plaques. ${ }^{4}$ A recent whole blood flow cytometry pilot study identified significantly higher platelet surface CD40 ligand expression in patients with acute symptomatic compared with asymptomatic moderate $(>50 \%)$ carotid stenosis, without an increase in expression of other markers of platelet activation. ${ }^{5}$ To our knowledge, no previous study has compared the platelet activation status of asymptomatic severe $(\geqslant 70 \%)$ carotid stenosis patients with that of both acute and convalescent symptomatic severe carotid stenosis patients. This information could improve our understanding of the mechanisms responsible for the higher risk of stroke in patients with symptomatic carotid stenosis and facilitate improved strategies for stroke prevention.

We carried out an observational analytical pilot study, using whole blood flow cytometry, to investigate whether there were differences in the platelet activation status of patients with acute or convalescent symptomatic and asymptomatic severe carotid stenosis. We hypothesised that we would find excessive platelet activation in patients with symptomatic compared with asymptomatic stenosis.

\section{METHODS \\ Patients}

Consecutive inpatients with symptomatic severe carotid stenosis, eligible for the study, were recruited from the Acute Brain Injury Unit at the National Hospital for Neurology and Neurosurgery, University College London Hospitals, and Atkinson Morley's Hospital, London. Consecutive outpatients with symptomatic or asymptomatic severe carotid stenosis, eligible for the study, were recruited from the Stroke Clinic at the National Hospital for Neurology and Neurosurgery, London, and from the vascular clinics at University College London Hospitals and Atkinson Morley's Hospital, London. One examiner (DJHM) clinically assessed all study patients; information about the method of detection of carotid stenosis in the asymptomatic group, stroke risk

Abbreviations: CDUS, colour Doppler ultrasound; FBC, full blood count; MPV, mean platelet volume; TIA, transient ischaemic attack 
factors, smoking status, alcohol intake, and medication use was collected prospectively. The local research ethics committees of the participating hospitals approved the study. Written informed consent (or written assent, where appropriate) was obtained in all cases.

Patients with symptomatic severe carotid stenosis were included in this group if they were $>18$ years of age; had an ischaemic stroke or TIA in the vascular territory supplied by a severe $(\geqslant 70 \%)$ ipsilateral carotid stenosis or occlusion within the preceding four weeks (acute phase); and were likely to be available for follow-up at least three months after symptom onset (convalescent phase). Patients with carotid occlusion were included only if no other cause for stroke or TIA was identified. Brain computed tomography (CT) or magnetic resonance imaging (MRI), and colour Doppler ultrasound (CDUS) examination of carotid and vertebral arteries were done in all patients. Patients were classified as having $>70 \%$ carotid stenosis if the peak systolic velocity in the internal carotid artery (PSV ICA) was greater than $2.1 \mathrm{~m} / \mathrm{s}$, the end diastolic velocity $\left(\right.$ EDV $\left._{\text {ICA }}\right)$ was greater than $1.1 \mathrm{~m} / \mathrm{s}$, or the ratio of the PSV $\mathrm{ICA}_{\mathrm{ICA}}$ to the PSV in the common carotid artery was greater than 4.0. ${ }^{6}$ Patients with carotid stenosis on CDUS also underwent extracranial magnetic resonance angiography (MRA) to further quantify the degree of carotid stenosis. If the results of CDUS and MRA examinations were discordant, and the patient was a potential candidate for carotid endarterectomy or endovascular treatment, intra-arterial catheter angiography was performed to quantify the degree of carotid stenosis.

We excluded patients with a history of myocardial infarction, major surgery, or systemic haemorrhage within the preceding three months; primary intracerebral haemorrhage; ongoing unstable angina; unstable symptomatic peripheral vascular disease; or if they had an active infection, vasculitis, neoplasia, or known bleeding or clotting diathesis.

Patients with asymptomatic severe carotid stenosis had $\geqslant 70 \%$ stenosis of the internal or common carotid artery on CDUS that was not associated with stroke or TIA in that or another vascular territory within the preceding three years. Some of these patients also underwent extracranial MRA. One patient had asymptomatic severe carotid restenosis noted on follow up CDUS examination five years after undergoing balloon angioplasty without stenting for asymptomatic severe atherosclerotic carotid stenosis. Eight patients had a history of contralateral symptomatic severe carotid stenosis requiring endarterectomy or endovascular treatment in the past. However, none had symptoms of stroke or TIA for at least the preceding three years, and all had $<70 \%$ stenosis of the treated carotid artery on follow up CDUS. The exclusion criteria were the same as those applied to the symptomatic group.

\section{Blood sampling and laboratory tests}

All subjects were rested for at least 20 minutes. A tourniquet was applied to the arm and careful atraumatic venepuncture carried out in all cases. Blood was collected from a free flowing vein via a sterile 21 gauge Butterfly needle (Venisystems, Abbott, Ireland) and a Vacutainer system with a Luer adaptor (Becton Dickinson Vacutainer Systems, UK). For all studies, the tourniquet was released during collection of the first $4 \mathrm{ml}$ of blood into a sterile Vacutainer tube containing $7.2 \mathrm{mg}$ of $\mathrm{K}_{2}$ EDTA or $0.054 \mathrm{ml}$ of $15 \% \mathrm{~K}_{3}$ EDTA, and this sample was used for measurement of a full blood count (FBC), including measurement of the mean platelet volume (MPV) with a GEN-S haematology analyser (Beckman Coulter United Kingdom Ltd, High Wycombe, UK). Two further $4.5 \mathrm{ml}$ samples were collected into Vacutainer tubes containing $0.5 \mathrm{ml}$ of $3.2 \%$ buffered sodium citrate. The first citrate-anticoagulated sample was used for whole blood flow cytometric analysis (see below), and the other for measurement of the MPV and platelet count in citrate-anticoagulated blood.

The flow cytometer used in this study was a Coulter EPICS XL-MCL (Beckman Coulter United Kingdom Ltd, UK), and the method used was adapted from the flow cytometric protocol that has been described previously ${ }^{7}$ and validated in our laboratory. ${ }^{8} 9$ Platelets were distinguished from red and white blood cells by their characteristic pattern of forward scatter (FS) and side scatter (SS), which depend on their size and granularity, respectively. ${ }^{10} \mathrm{~A}$ gate was manually positioned around the platelet cloud on the "log FS" versus "log SS" histogram, the gating settings were saved on the flow cytometer, and 10000 platelet events were analysed. To confirm that the majority of cells within the gate in the test sample were platelets, the percentage binding of an "activation-independent" platelet-specific monoclonal antibody to the GpIb $\alpha$ subunit of the GpIb-IX-V complex was calculated (see Results). ${ }^{11}$ CD62P (P-selectin, GMP-140, PADGEM) is only expressed on the platelet surface after $\alpha$ or dense granule secretion, and CD63 (granulophysin) is expressed on the platelet surface after the release of lysosomes or dense granules. ${ }^{12}{ }^{13}$ Platelet activation leads to a conformational change in the GpIIb/IIIa receptor that facilitates ligand binding and mediates platelet-platelet aggregation. PACl is an IgM monoclonal antibody that appears to bind specifically to the GpIIb/IIIa binding site exposed during platelet activation. ${ }^{11}$ Whole blood flow cytometry was used to assess platelet activation status by quantifying the percentage platelet surface expression of CD62P and CD63, and PACl binding. ${ }^{8} 9$

Platelet surface CD62P mediates the adhesion of platelets to leucocytes. ${ }^{14}$ The percentages of neutrophil-platelet, monocyte-platelet, and lymphocyte-platelet complexes were also quantified by flow cytometry as additional markers of platelet activation using previously described methods. ${ }^{89}$ For these assays, $5 \mu \mathrm{l}$ aliquots of anti-IgG $\mathrm{I}_{1}$-PE control antibody and anti-CD42b-PE antibody were put into the isotype control sample tube and test sample tube, respectively. Then $5 \mu \mathrm{l}$ of anti-CD45-R-phycoerythrin-cyanine 5 (RPECy5) (a pan-leucocyte marker), followed by $65 \mu \mathrm{l}$ of HEPES buffered saline, was added to both tubes, the samples gently mixed and covered until use. Within five minutes of venepuncture, a $25 \mu \mathrm{l}$ aliquot of whole blood was put into both the control and test tubes, and the samples incubated at room temperature for 10 minutes. Then the samples were fixed with $84 \mu \mathrm{l}$ of diluent fixative (made up of $0.5 \mathrm{ml}$ of $10 \%$ formaldehyde solution, $0.6 \mathrm{ml}$ of $10 \times$ Hanks Balanced Saline Solution without calcium, magnesium, or phenol red (Gibco BRL, Life Technologies, Paisley, UK) and $0.9 \mathrm{ml}$ of distilled water). After a further 10 minute incubation period, $840 \mu \mathrm{l}$ of distilled water was added to each tube in order to induce erythrocyte lysis. The samples were analysed on the flow cytometer within three hours of venepuncture. We used a low "flow rate" to minimise the possibility of detecting dual events-that is, the simultaneous passage of a single leucocyte and a single unattached platelet through the flow chamber. A minimum of 1000 monocyte events were analysed in each assay, and the percentages of neutrophils, monocytes, and lymphocytes complexed to platelets were calculated.

\section{Statistical methods}

We used the Mann-Whitney U test for comparison of median values, an unpaired $t$ test for comparison of mean values, and the $\chi^{2}$ test for comparison of proportions between the asymptomatic and acute and convalescent symptomatic groups. We used Wilcoxon's signed rank test to compare median values, and a paired $t$ test to compare mean values 
Table 1 Clinical characteristics of the patients in the present study

\begin{tabular}{|c|c|c|c|}
\hline & $\begin{array}{l}\text { Asymptomatic } \\
\text { severe } \\
\text { carotid stenosis }\end{array}$ & $\begin{array}{l}\text { Acute symptomatic } \\
\text { severe carotid } \\
\text { stenosis }\end{array}$ & $\begin{array}{l}\text { Convalescent } \\
\text { symptomatic severe } \\
\text { carotid stenosis }\end{array}$ \\
\hline Number & 16 & 19 & 16 \\
\hline Mean age (years) & 72 & 69 & 68 \\
\hline $\operatorname{Sex}(M / F)$ & $11 / 5$ & $9 / 10$ & $8 / 8$ \\
\hline \multicolumn{4}{|l|}{ Antithrombotic therapy } \\
\hline ASA monotherapy & 14 & 11 & 8 \\
\hline$A S A+D P$ & 2 & 5 & 3 \\
\hline $\mathrm{ASA}+\mathrm{DP}+$ dalteparin & 0 & 0 & 1 \\
\hline ASA + dalteparin & 0 & 1 & 0 \\
\hline ASA + azapropazone & 0 & 1 & 1 \\
\hline clopidogrel & 0 & 1 & 1 \\
\hline Warfarin & 0 & 0 & 1 \\
\hline None of above & 0 & 0 & $1^{*}$ \\
\hline Prior stroke/transient ischaemic attack, $n(\%)$ & $10(62.5)$ & $12(63.2)$ & $9(56.3)$ \\
\hline Ischaemic heart disease, n (\%) & $9(56.3)$ & $8(42.1)$ & $6(37.5)$ \\
\hline Hypertension, n (\%) & $14(87.5)$ & $17(89.5)$ & $16(100)$ \\
\hline Diabetes mellitus, $n(\%)$ & $4(25.0)$ & $3(15.8)$ & $2(12.5)$ \\
\hline Peripheral vascular disease, $\mathrm{n}(\%)$ & $8(50.0)$ & $6(31.6)$ & $5(31.3)$ \\
\hline Atrial fibrillation/flutter & 0 & 0 & 0 \\
\hline Family history of stroke, $n(\%)$ & $5(31.3)$ & $7(36.8)$ & $5(31.3)$ \\
\hline Prior venous thrombosis, $\mathrm{n}(\%)$ & $0(0)$ & $1(5.3)$ & $2(12.5)$ \\
\hline Migraine, $\mathrm{n}(\%)$ & $2(12.5)$ & $4(21.1)$ & $3(18.8)$ \\
\hline \multicolumn{4}{|l|}{ Smoking status, $\mathrm{n}(\%)$} \\
\hline Current & $3(18.8)$ & $4(21.1)$ & $2(12.5)$ \\
\hline Ex-smoker & $8(50.0)$ & $8(42.1)$ & $8(50.0)$ \\
\hline Non-smoker & $5(31.3)$ & $7(36.8)$ & $6(37.5)$ \\
\hline Alcohol intake (median units/week) & 1 & 0.5 & 0 \\
\hline
\end{tabular}

between acute and convalescent symptomatic patients who had matched samples in both phases after symptom onset; $\mathrm{p}<0.05$ was considered statistically significant. All statistical calculations were carried out with SPSS-10 for Windows or Microsoft Excel 97 data analysis software.

\section{RESULTS}

Nineteen patients with symptomatic severe carotid stenosis were assessed in the acute phase (0-21 days), and 16 of these were reassessed in the convalescent phase (79-365 days) after ischaemic stroke or TIA. Eight patients were reassessed at least three months after successful carotid endarterectomy or endovascular treatment. Of the remaining eight patients, four were reassessed before endarterectomy or endovascular treatment, two patients had carotid occlusion from the outset, and two were deemed unsuitable for surgery or endovascular treatment. One patient in the convalescent phase was reassessed 79 days after presentation, before undergoing endovascular treatment, and one patient was assessed at 86 days after symptom onset because she was emigrating. All remaining patients were reassessed at least 90 days after symptom onset, as originally planned. Three patients did not have repeat laboratory testing during the convalescent phase; one patient died, one moved away, and one declined further investigations. The results were compared with those obtained from 16 patients with asymptomatic severe carotid stenosis.

The clinical characteristics of the patients are summarised in table 1 . The vascular risk factor profiles were similar in all groups. The median internal carotid artery (ICA) stenosis on CDUS was $80-95 \%$ in acute symptomatic, $70-79 \%$ in convalescent symptomatic, and $80-95 \%$ in asymptomatic patients. The median ICA stenosis remained elevated in the convalescent symptomatic group despite successful treatment of the stenosing plaque in $8 / 16$ patients because of the persistence of severe stenosis or occlusion in the remaining eight patients at the time of re-testing. Most patients were receiving aspirin at the time of assessment. Patients with symptomatic stenosis were on variable doses of aspirinbetween $75 \mathrm{mg}$ and $300 \mathrm{mg}$ daily (median daily dose = $150 \mathrm{mg}$ in the acute phase and $75 \mathrm{mg}$ in the convalescent phase). Asymptomatic patients received $37.5 \mathrm{mg}$ to $300 \mathrm{mg}$ of aspirin daily (median daily dose $=150 \mathrm{mg}$ ). However, treatment with aspirin and modified release dipyridamole (200 mg twice daily), or clopidogrel (75 mg daily), or anticoagulation were more commonly used in patients with symptomatic stenosis.

The mean platelet count in EDTA was higher in the acute $\left(275 \times 10^{9} / \mathrm{l}, \quad \mathrm{p}=0.007\right)$ and convalescent symptomatic $\left(266 \times 10^{9} / \mathrm{l}, \mathrm{p}=0.04\right)$ than in the asymptomatic patients $\left(223 \times 10^{9} / 1\right)$. The mean platelet count in citrate was also higher in acute $\left(231 \times 10^{9} / \mathrm{l}, \mathrm{p}=0.05\right)$ and convalescent symptomatic $\left(239 \times 10^{9} / \mathrm{l}, \mathrm{p}=0.02\right)$ than in asymptomatic patients $\left(193 \times 10^{9} / 1\right)$. Otherwise, there were no significant differences in any FBC variables between the symptomatic and asymptomatic groups.

The median percentage GpIb expression was similar in acute $(99.1 \%)$ and convalescent symptomatic (99.2\%) and asymptomatic patients $(99.3 \%)$, confirming that the majority of cells analysed on the flow cytometer in each group were platelets. There were no significant differences in the median percentage platelet expression of CD62P and CD63, or PAC1 binding between the acute or convalescent symptomatic and asymptomatic patients (fig 1).

The median percentage of neutrophil-platelet $(\mathrm{p}=0.004$, fig $2 \mathrm{~A})$, monocyte-platelet $(\mathrm{p}=0.046$, fig $2 \mathrm{~B})$, and lymphocyte-platelet complexes ( $p=0.02$, fig $2 \mathrm{C}$ ) was higher in acute symptomatic than asymptomatic patients. In the convalescent phase, the median percentages of all leucocyte-platelet complexes in the symptomatic group had dropped to levels similar to those found in the asymptomatic group $(p \geqslant 0.07$, see fig 2). Among the patients with symptomatic stenosis for whom matched data in both phases after symptom onset were available, there were no significant differences between 

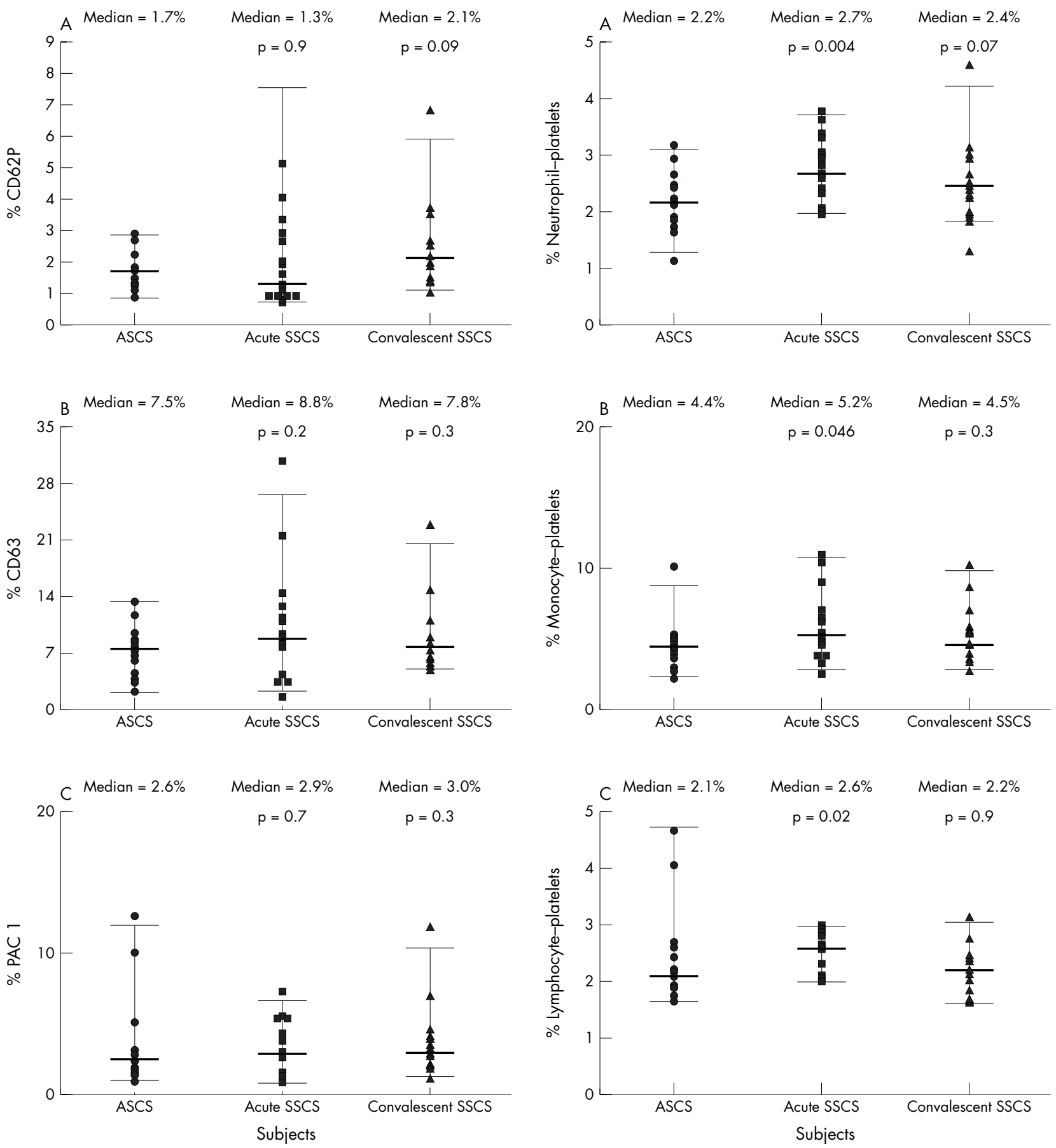

Figure 1 Comparison of (A) \% CD62P expression, (B) \% CD63 expression, and (C) \% PACl binding between patients with asymptomatic severe carotid stenosis (ASCS) and acute and convalescent symptomatic severe carotid stenosis (SSCS) patients. Symbols, individual values; bold horizontal lines, median values; vertical bars, 5th to 95th percentiles.

the acute and convalescent samples $(p \geqslant 0.1)$ in the percentage expression of any of the platelet activation markers or the percentage of circulating leucocyte-platelet complexes.

The use of different antithrombotic regimens (see table 1) could also have influenced the observed differences between the groups. Therefore, we compared data obtained from the acute symptomatic subgroup with the asymptomatic patients on aspirin monotherapy. The mean percentage of neutrophilplatelet complexes $(2.7 \% \vee 2.2 \%, \mathrm{p}=0.03)$ and the median

Figure 2 Comparison of (A) \% neutrophil-platelet complexes, (B) \% monocyte-platelet complexes, and (C) \% lymphocyte-platelet complexes between patients with asymptomatic severe carotid stenosis (ASCS) and acute and convalescent symptomatic severe carotid stenosis (SSCS) patients. Symbols, individual values; bold horizontal lines, median values; vertical bars, 5 th to 95 th percentiles.

percentage of monocyte-platelet complexes (5.4\% $v 4.4 \%$, $\mathrm{p}=0.03)$ was higher in acute symptomatic $(\mathrm{n}=11)$ than asymptomatic patients on aspirin only $(\mathrm{n}=14)$. However, the median percentage of lymphocyte-platelet complexes was not significantly higher in acute symptomatic patients on aspirin only $(2.3 \% v 2.1 \% \mathrm{p}=0.2)$. Platelet counts in EDTAanticoagulated $(\mathrm{p}=0.02)$ and citrate-anticoagulated blood $(p=0.04)$ were higher in acute symptomatic than asymptomatic patients on aspirin only. Otherwise, differences 
between the symptomatic and asymptomatic patients on aspirin were not significant for any variable measured.

\section{DISCUSSION}

We found an increased mean platelet count and increased leucocyte-platelet complex formation in patients with acute symptomatic compared with asymptomatic severe carotid stenosis. These indicators of increased platelet activation may contribute to the early excess risk of stroke in patients with recently symptomatic severe carotid stenosis. Previous studies have shown that the platelet count may be increased, ${ }^{15}$ decreased, ${ }^{16}$ or remain unchanged ${ }^{17}$ in the acute phase after cerebral infarction compared with controls. However, to our knowledge, platelet counts have not been investigated in acute symptomatic versus asymptomatic severe carotid stenosis.

The absence of significant differences in CD62P expression between patients with acute symptomatic and asymptomatic carotid stenosis is not incompatible with our findings of increased leucocyte-platelet complexes in the acute symptomatic group, because CD62P may be rapidly shed from the surface of circulating degranulated platelets. ${ }^{10}$ Recent studies in patients with ischaemic heart disease have shown that an increase in the percentage of monocyte-platelet complexes may be a more sensitive indicator of in vivo platelet activation than an increase either in the percentage of neutrophilplatelet complexes ${ }^{18}$ or CD62P expression on platelets. ${ }^{18}{ }^{19}$ Our results are consistent with this hypothesis, because the absolute percentages of monocyte-platelet complexes were higher than the absolute percentages of neutrophil-platelet complexes in individual patients (see fig $2 \mathrm{~A}, \mathrm{~B}$ ).

The higher percentage of leucocyte-platelet complexes in acute symptomatic compared with asymptomatic patients may represent "primary" differences in platelet activation status between the groups. These findings could also be "secondary" to carotid plaque activation in the symptomatic group, with subsequent platelet activation and leucocyteplatelet complex formation, or might have arisen as an acute phase response to cerebral or ocular ischaemia or infarction. It is possible that excessive leucocyte-platelet complex formation represents both a "primary" and "secondary" phenomenon in patients with acute symptomatic stenosis. Alternatively, excessive leucocyte activation may have contributed to the higher percentage of leucocyte-platelet complexes in patients with acute symptomatic stenosis. ${ }^{80}$ However, the finding of an increased platelet count in acute and convalescent symptomatic compared with asymptomatic patients suggests that there is an ongoing stimulus to platelet production, and possibly platelet activation, in patients with symptomatic stenosis. Additional prospective studies are required to determine whether patients with severe carotid stenosis and excessive leucocyte-platelet complex formation are at higher risk of subsequent stroke or TIA than patients with lower percentages of circulating complexes. It would also be of interest to examine the relationship between platelet activation status and plaque morphology to determine whether patients with lipid laden echolucent plaques have excessive platelet activation compared with patients with echodense fibrous plaques. If this were the case, more potent antithrombotic therapy in these high risk patients, or better selection of patients for endarterectomy or endovascular treatment, may facilitate more effective stroke prevention.

Increased leucocyte-platelet complexes may play a role in the pathogenesis of TIA or stroke in patients with recently symptomatic carotid stenosis. Platelets and leucocytes may have stimulatory influences on one another, and leucocyteplatelet complex formation is likely to facilitate interaction between the two cell types..$^{21}$ When an atherosclerotic plaque ruptures, platelet adhesion to the plaque contents may stimulate further platelet activation and neutrophil-platelet complex formation. This may, in turn, amplify platelet secretion and aggregation in the thrombogenic milieu of the plaque, ${ }^{21}$ and increase the risk of platelet thromboembolism. Furthermore, neutrophil-platelet complex formation may promote neutrophil activation, with the release of inflammatory cytokines ${ }^{21}$ that could attract other leucocytes to the ruptured plaque. The percentage of monocyte-platelet complexes was higher in acute symptomatic than asymptomatic patients, and monocyte-platelet complexes were also elevated in the subgroup of acute symptomatic patients on aspirin only. These data are interesting because platelets may supply cholesterol to monocytes, ${ }^{22}$ which may mature into lipid laden macrophages. This may be important in the pathogenesis of ischaemic infarction, because atherosclerotic plaques that rupture and cause symptoms have a higher lipid content and larger numbers of macrophages and inflammatory cells than those that do not rupture. ${ }^{4}$ Furthermore, platelet $\mathrm{CD} 62 \mathrm{P}$ has been shown to induce tissue factor expression on monocytes, ${ }^{23}$ which could, in turn, lead to activation of the coagulation pathway and increase the risk of thromboembolism. Because some of the differences between the groups in this study just reached statistical significance, and because there are marked differences in the risk of stroke between recently symptomatic and asymptomatic patients, further studies with larger groups of patients are required to confirm our findings, and to investigate other potential haemostatic factors that might contribute to the pathogenesis of stroke in patients with severe carotid stenosis.

We did not find an increase in CD63 expression or PACl binding in symptomatic compared with asymptomatic patients overall, but the small number of patients assessed in this study may have contributed to a type II error. It is not surprising that there were no significant differences in platelet activation markers between the convalescent symptomatic and asymptomatic patients. This may be explained, in part, by resolution of the acute phase response in the three month period after stroke or TIA, in combination with some degree of plaque healing before repeat venepuncture. Furthermore, successful treatment of the stenosing plaque in $8 / 16$ patients with symptomatic stenosis before re-testing during the convalescent phase undoubtedly reduced the degree of shear induced platelet activation compared with the acute setting.

Overall, this study improves our understanding of the potential mechanisms involved in the pathogenesis of stroke or TIA in patients with severe carotid stenosis. Further work is required to examine the effects of different antiplatelet regimens on ex vivo platelet activation in this patient population.

\section{ACKNOWLEDGEMENTS}

We thank Diana Colquhoun for performing some of the colour Doppler ultrasound examinations, and all consultant staff at the National Hospital for Neurology and Neurosurgery, University College London Hospitals, and Atkinson Morley's Hospital, London who allowed us to study their patients. We also thank all of the subjects who generously gave up their time to participate in this study.

\section{Authors' affiliations}

D J H McCabe, P S Sidhu, M M Brown, Stroke Research Group, Institute of Neurology, National Hospital for Neurology and Neurosurgery, University College London, London, UK

D J H McCabe, P Harrison, I J Mackie, G Purdy, A S Lawrie, S J Machin, The Haemostasis Research Unit, Department of Haematology, University College London, London, UK

H Watt, The Dementia Research Group, Institute of Neurology, National Hospital for Neurology and Neurosurgery, and Medical Statistics Unit, London School of Hygiene and Tropical Medicine, London, UK 
P Harrison, Oxford Haemophilia Centre and Thrombosis Unit, Churchill Hospital, Oxford, UK

Dr McCabe was funded by a grant from the Brain Research Trust, England. Professor Brown's Chair in Stroke Medicine is supported by The Reta Lila Weston Trust for Medical Research, U.K. The ultrasound laboratory at Atkinson Morley's Hospital was funded by the Wellcome Trust and the Neurosciences Research Foundation.

Competing interests: none declared

The 21 gauge Butterfly needles were purchased from Venisystems, Abbott, Ireland, and the Vacutainer system and Luer adaptors from Becton Dickinson Vacutainer Systems, UK. The anti-lgM-fluorescein isothiocyanate (FITC)-conjugated isotype control mouse monoclonal antibody used in the PACl assays was purchased from Sigma-Aldrich Inc., St Louis, USA, and the anti-PACl-FITC-conjugated IgMK mouse monoclonal test antibody was purchased from Becton Dickinson, San Jose, USA. The anti-CD45-R-phycoerythrin-cyanine 5 (RPE-Cy5) antibody used in the leucocyte-platelet complex assays was purchased from Dako, Glostrup, Denmark. All other mouse monoclonal antibodies used in the flow cytometry studies were purchased from Immunotech, Beckman Coulter, Marseille, France.

\section{REFERENCES}

1 The European Carotid Surgery Trialists' Collaborative Group. Risk of stroke in the distribution of an asymptomatic carotid artery. Lancet 1995;345:209-12.

2 Executive Committee for the Asymptomatic Carotid Atherosclerosis Study. Endarterectomy for asymptomatic carotid artery stenosis. JAMA 1995;273:1421-8.

3 North American Symptomatic Carotid Endarterectomy Trial Collaborators. Beneficial effect of carotid endarterectomy in symptomatic patients with highgrade carotid stenosis. N Engl J Med 1991;325:445-53.

4 Golledge J, Greenhalgh RM, Davies AH. The symptomatic carotid plaque. Stroke 2000;31:774-81.

5 Cha JK, Jeong MH, Jang JY, et al. Serial measurement of surface expressions of CD63, P-selectin and CD40 ligand on platelets in atherosclerotic ischaemic stroke. A possible role of CD40 ligand on platelets in atherosclerotic ischaemic stroke. Cerebrovasc Dis 2003;16:376-82.

6 Moneta GL, Edwards JM, Chitwood RW, et al. Correlation of North American Symptomatic Carotid Endarterectomy Trial (NASCET) angiographic definition of $70 \%$ to $99 \%$ internal carotid artery stenosis with duplex scanning. J Vasc Surg 1993;17:152-7.
7 Shattil SJ, Cunningham M, Hoxie JA. Detection of activated platelets in whole blood using activation-dependent monoclonal antibodies and flow cytometry. Blood 1987;70:307-15.

8 Joseph JE, Harrison P, Mackie IJ, et al. Increased circulating platelet-leucocyte complexes and platelet activation in patients with antiphospholipid syndrome, systemic lupus erythematosus and rheumatoid arthritis. Br J Haematol 2001;115:451-9.

9 McCabe DJ, Harrison P, Mackie IJ, et al. Platelet degranulation and monocyteplatelet complex formation are increased in the acute and convalescent phases after ischaemic stroke or transient ischaemic attack. $\mathrm{Br} J$ Haematol 2004; 125:777-87.

10 Michelson AD. Flow cytometry: a clinical test of platelet function. Blood 1996;87:4925-36

11 Abrams C, Shattil SJ. Immunological detection of activated platelets in clinical disorders. Thromb Haemost 1991;65:467-73.

12 Grau AJ, Ruf A, Vogt A, et al. Increased fraction of circulating activated platelets in acute and previous cerebrovascular ischemia. Thromb Haemost 1998;80:298-301.

13 Israels SJ, Gerrard JM, Jacques YV, et al. Platelet dense granule membranes contain both granulophysin and P-selectin (GMP-140). Blood 1992;80:143-52.

14 de Bruijne-Admiraal LG, Modderman PW, Von dem Borne AEGKr, et al. P-selectin mediates $\mathrm{Ca}(2+)$-dependent adhesion of activated platelets to many different types of leucocytes: detection by flow cytometry. Blood 1992;80:134-42.

15 Numminen H, Hillbom M, Juvela S. Platelets, alcohol consumption, and onset of brain infarction. J Neurol Neurosurg Psychiatry 1996;61:376-80.

16 O'Malley T, Langhorne P, Elton RA, et al. Platelet size in stroke patients. Stroke 1995:26:995-9.

17 Butterworth RJ, Bath PMW. The relationship between mean platelet volume, stroke subtype and clinical outcome. Platelets 1998;9:359-64.

18 Michelson AD, Barnard MR, Krueger LA, et al. Circulating monocyte-platelet aggregates are a more sensitive marker of in vivo platelet activation than platelet surface P-selectin: studies in baboons, human coronary intervention, and human acute myocardial infarction. Circulation 2001;104:1533-7.

19 Furman MI, Barnard MR, Krueger LA, et al. Circulating monocyte-platelet aggregates are an early marker of acute myocardial infarction. J Am Coll Cardiol 2001;38:1002-6.

20 Akopov SE, Simonian NA, Grigorian GS. Dynamics of polymorphonuclear leucocyte accumulation in acute cerebral infarction and their correlation with brain tissue damage. Stroke 1996;27:1739-43.

21 Li N, Hu H, Lindqvist $M$, et al. Platelet-leucocyte cross talk in whole blood. Arterioscler Thromb Vasc Biol 2000;20:2702-8.

22 Mendelsohn ME, Loscalzo J. Role of platelets in cholesteryl ester formation by U-937 cells. J Clin Invest 1988;81:62-8.

23 Celi A, Pellegrini G, Lorenzet R, et al. P-selectin induces the expression of tissue factor on monocytes. Proc Natl Acad Sci U S A 1994;91:8767-71. 